\title{
Oxacillin (Methicillin) Resistant Staphylococci in Domestic Animals in the Czech Republic
}

\author{
Jaroslav Bzdil ${ }^{1}$, Monika Zouharova ${ }^{2}$, Katerina Nedbalcova ${ }^{2}$, Vladimir Sladecek ${ }^{1}$, David Senk ${ }^{1}$ \\ and Ondrej Holy ${ }^{3, *}$ \\ 1 Ptacy s.r.o., Valasska Bystrice 194, 75627 Valasska Bystrice, Czech Republic; vetmed@seznam.cz (J.B.); \\ sladecek.vladimir@gmail.com (V.S.); ptacy@ptacy-sro.cz (D.S.) \\ 2 Department of Infectious Diseases and Preventive Medicine, Veterinary Research Institute Brno, \\ Hudcova 296/70, 62100 Brno, Czech Republic; monika.zouharova@vri.cz (M.Z.); \\ katerina.nedbalcova@vri.cz (K.N.) \\ 3 Science and Research Centre, Faculty of Health Sciences, Palacky University Olomouc, Hnevotinska 3, \\ 77515 Olomouc, Czech Republic \\ * Correspondence: ondrej.holy@upol.cz; Tel.: +420-585632818
}

check for updates

Citation: Bzdil, J.; Zouharova, M.; Nedbalcova, K.; Sladecek, V.; Senk, D.; Holy, O. Oxacillin (Methicillin) Resistant Staphylococci in Domestic Animals in the Czech Republic. Pathogens 2021, 10, 1585. https:// doi.org/10.3390/pathogens10121585

Academic Editor: Rachel McLoughlin

Received: 20 November 2021

Accepted: 30 November 2021

Published: 6 December 2021

Publisher's Note: MDPI stays neutral with regard to jurisdictional claims in published maps and institutional affiliations.

Copyright: (c) 2021 by the authors. Licensee MDPI, Basel, Switzerland. This article is an open access article distributed under the terms and conditions of the Creative Commons Attribution (CC BY) license (https:// creativecommons.org/licenses/by/ $4.0 /)$.

\begin{abstract}
The aim of this study was to describe the prevalence of different Staphylococcus species isolated from pathological processes and lesions in domestic animals in the Czech Republic and to detect and describe oxacillin (methicillin)-resistant strains (MRS). During the years 2019-2020, a total of 5218 veterinary clinical samples from the Czech Republic were tested. Testing was performed by culture methods and typing by molecular phenotypic methods MALDI-TOF MS and PCR. Antimicrobial susceptibility testing of the strains was performed by the disk diffusion method. A total of 854 staphylococci strains were identified (16.37\% prevalence), out of which 43 strains of 6 species of staphylococci were MRS ( $n=43 ; 0.82 \%$ prevalence). Of the MRS strains, the most prevalent species were Staphylococcus pseudintermedius ( $n=24 ; 0.46 \%$ prevalence) and Staphylococcus aureus ( $n=7 ; 0.13 \%$ prevalence). Susceptibility testing showed resistance to beta-lactam antibiotics and, depending on the species, also to trimethoprim/sulfamethoxazole, gentamicin, tetracycline, erythromycin, clindamycin, and enrofloxacin. For further characterization of MRS, PCR assay for virulence factor genes was performed. Seven of the 14 target genes were observed only in S. aureus, except for the eno gene encoding laminin-binding protein, which was also detected in other staphylococci. It is necessary to emphasize the issue of correct using of antimicrobials in practice and antibiotic policy in university teaching and to create stricter legislation that would prevent the widespread use of antimicrobials in veterinary medicine, especially in livestock to reduce the emergence and spread of antimicrobial resistance.
\end{abstract}

Keywords: prevalence; occurrence; organs; virulence genes; susceptibility; veterinary medicine

\section{Introduction}

Several studies have shown that Gram-positive bacteria are apparently the most common microorganisms isolated from different human and veterinary clinical materials [1-5]. This group of bacteria includes staphylococci, which are mostly commensals of the skin and mucous membranes in animals and humans [6,7]. Many of them are opportunistic pathogens causing pyogenic infections [6,8]. Of the coagulase-positive staphylococci (CPS), the species of special human and veterinary importance is Staphylococcus aureus, which causes local purulent and systemic infections, as well as human and animal toxaemia [5,9-11]. Other CPS such as Staphylococcus pseudintermedius, Staphylococcus intermedius, and Staphylococcus delphini, which are included in the Staphylococcus intermedius-group, as well as Staphylococcus schleiferi ssp. coagulans and S. hyicus, are significant primarily in the veterinary sphere $[6,12-14]$. 
Coagulase-negative staphylococci (CNS) have been considered a component of normal microbiota but can also act as human and animal pathogens due to their invasiveness, production of toxins [6,13], adhesins and hemolysins, and the ability to form biofilms $[1,3,7]$. Members of this group can cause a wide range of animal and human diseases, namely, local infections of skin, mucous membranes of urinary and respiratory apparatus, and mammary gland, and they have been reported as a potential cause of septicaemia in human and animals $[2,9,13,15,16]$. Due to biofilm formation, some CNS strains (for example, S. epidermidis) can also cause foreign body-associated infections [17]. Some Staphylococcus haemolyticus strains can cause infections in debilitated dialysis patients, diabetic patients, and patients after surgery, and it is well known for its ability to develop multidrug-resistant forms in bedridden patients [18]. Staphylococci as a zoonotic agent and a source of resistance and pathogenicity genes for people are a current topic of the present time. Many recent works have described different cases of human infections caused by animal strains of staphylococci or food-born staphylococci strains [8,11,19-21].

Varying levels of antimicrobial resistance are found in human and veterinary strains of staphylococci. Regarding therapy, methicillin-resistant strains of staphylococci need special attention because they show co-resistance to other beta-lactam antibiotics including oxacillin/methicillin and, in many cases, also to aminoglycosides, tetracyclines, macrolides, chloramphenicol, fluoroquinolones, and rifampicin [9].

Oxacillin resistance is encoded by the mec $A$ gene [9] and its two homologues, mecB and mecC, with several alotypes. As known for mecA, the gene homolog mecC is also not restricted to $S$. aureus, but found in several staphylococcal species including $S$. sciuri, S. stepanovicii, and S. xylosus (mecC1 allotype). First investigations showed a wide geographical distribution of mecC-MRSA in Europe and a broad diversity of host species including livestock, companion, and wildlife animals. In particular, wild rodents and insectivores might serve as a reservoir for staphylococci harboring mecC [22]. Despite the fact that methicillin resistance has no particular effect on the clinical course of disease, it can fundamentally affect the antimicrobial therapeutic effect and, even worse, may be a source of resistance genes for other S. aureus strains in other animals and humans [23]. In human medicine, methicillin-resistant $S$. aureus (MRSA) is often detected among the hospital-associated methicillin resistant Staphylococcus aureus (HA-MRSA) strains [9] and, in veterinary medicine, staphylococcal resistance to methicillin (oxacillin) was detected, particularly in cows with mastitis or in skin lesion samples from cats and dogs [12,23-25].

Staphylococci can express a wide range of virulence factors including surface proteins, exoenzymes, and extracellular toxins that allow it to adhere to biotic or abiotic surfaces, invade or avoid the immune system, and cause harmful toxic effects to the host. Ownership of these factors may strongly influence the course and severity of infection [26].

The first step of infection is bacterial adhesion to host extracellular matrix and plasma proteins. It is mediated by different proteins of the family of MSCRAMMs (microbial surface components recognizing adhesive matrix molecules) [27]. These molecules include EbpS (elastin-binding protein), Eno (laminin-binding protein), Cna (collagen-binding protein), Fib (fibrinogen-binding protein), and Bbp (bone sialoprotein-binding protein). The $F n b A$ and $F n b B$ proteins bind to fibronectin and fibrinogen, while clumping factors $C l f A$ and $C l f B$ bind to fibrinogen and promote bacterial adhesion to thrombi. Binding of these surface proteins to various substances present in the extracellular matrix allows the bacterium to invade host tissues [28].

Another very important step is accumulation of bacteria in multi-layered cell clusters. Such biofilm formation protects microorganisms from opsonophagocytosis and antimicrobial agents [29]. Biofilm formation in isolates occurs through the polysaccharide intercellular adhesin (PIA). The intracellular adhesion (ica) operon is essential for the control of biofilm production. The $i c a A D B C$ operon encodes three membrane proteins (IcaA, IcaD, and IcaC) with enzymatic activity and one extracellular protein (IcaB). The PIA, encoded by this operon also plays an important role in adhesion to epithelial cells and allows for escaping the immune system of the host [29]. 
Staphylococci may have a number of other virulence factors that are involved in the pathogenesis of the disease. Some symptoms associated with $S$. aureus infection are caused by toxins, such as toxic shock syndrome toxin 1 (TSST-1), enterotoxins, and exfoliative toxins (ETs). More than 20 SEs have been identified to date and are one of the most frequent causes of food-borne disease. TSST-1 causes a serious illness with high mortality. Exfoliative toxins (ETs) (also known as "epidermolytic" toxins) cause staphylococcal scalded skin syndrome (SSSS) characterized by destruction of desmosomal cell attachments resulting in detachment of the epidermis [9,30] (Votava et al. 2006; Oliveira et al. 2018). Some strains of $S$. aureus can also produce Panton-Valentine leucocidin $[4,16]$.

The presence of virulence factors in different staphylococcal strains varies depending on the location of the infection and the degree of virulence.

The aim of the present study was to describe species distribution of staphylococci isolated from pathological processes and lesions in domestic animals in the Czech Republic and to detect and characterize oxacillin/methicillin-resistant staphylococcal (MRS) strains, including their prevalence, site of infection, host specificity, and virulence factor determination.

\section{Material and Methods}

\subsection{Isolation and Identification of Bacteria: Bacteriological Confirmation}

A total of 5218 clinical samples from pathological processes and lesions of domestic animals between April 2019 and June 2020 underwent microbiological cultivation at the Veterinary Research Institute Brno (Czech Republic). Sampling was performed by instructed private veterinarians. Solid and slurry materials were sterile collected in $60 \mathrm{~mL}$ plastic containers (Dispolab Ltd. Brno, Czech Republic). Fluids were collected in $10 \mathrm{~mL}$ sterile closable tubes (Dispolab Ltd. Brno, Czech Republic). Swabs were taken using Transbak system containing Amies broth with active carbon (Dispolab Ltd. Brno, Czech Republic). All samples were stored and transported to the laboratory at $4{ }^{\circ} \mathrm{C}$.

\subsection{Samples from Digestive Tract}

Feces, rectal swabs, and swabs taken from the stomach lining were examined routinely by conventional methods of cultivation on meat peptone blood agar (MPBA) (Trios Ltd., Prague, Czech Republic), and plates were incubated aerobically at $37 \pm 1^{\circ} \mathrm{C}$ for $24 \mathrm{~h}$.

\subsection{Samples from the Skin; Urinary Apparatus; Oral Cavity; Eyes; Respiratory, Musculoskeletal} and Lymphatic Systems

The cultivation of hair; swabs and scrapings of skin; swabs of ear; urine and swabs of the urinary tract; swabs and the lavages of the respiratory tract, pharynx, conjunctiva and oral mucosa; the puncture of chest, lymph nodes and joints were performed on MPBA (Trios Ltd. Prague, Czech Republic), and the plates were again incubated aerobically at $37 \pm 1^{\circ} \mathrm{C}$ for $24 \mathrm{~h}$.

\subsection{Mammary Gland and Milk Samples}

The milk samples were again inoculated on MPBA (Trios Ltd., Prague, Czech Republic) after thorough shaking, and incubation was carried out aerobically at $37 \pm 1^{\circ} \mathrm{C}$ for $42-48 \mathrm{~h}$.

\subsection{Bacteriological Confirmation and Susceptibility Determination}

All types of colonies grown on plates were isolated, and suspected Gram-positive organisms were isolated and subsequently confirmed by the phenotypic molecular method using mass detector MALDI-TOF MS Microflex ${ }^{\mathrm{TM}}$ LT System (Bruker Daltonik GmbH, Bremen, Germany), on the basis of proteomics analyses and MALDI Biotyper software MBT Compass 4.1.100 (Bruker Daltonik GmbH, Bremen, Germany). In the plates with mixed bacterial cultures, the most prominent colony-forming unit agent was regarded as the leading pathogen. An identification score of 2.000 was set as the reliability threshold. The typing of strains with a lower score was specified by the MALDI-TOF method using a 
more exact library of spectra of the National Reference Laboratory for Staphylococci of the National Institute of Public Health in Prague or PCR method.

\subsection{Antimicrobial Susceptibility Testing}

Clinical strains were tested for antibiotics susceptibility by the disc diffusion method. The Mueller-Hinton agar (Trios Ltd., Prague, Czech Republic) and antibiotic discs were used for testing (Oxoid Ltd., Basingstoke, United Kingdom). The tested antibiotics were shown in Table 1 . The tests were assessed after $18-24 \mathrm{~h}$ of incubation at $37 \pm 1{ }^{\circ} \mathrm{C}$. The interpretation of values according to CLSI (2013), CLSI (2018), NCCLS (2002), EUCAST (2020), CASFM (2018), BD BBL (2020), and BIOPHARM (2020) standards was performed (see the Table 1) [31-37]. The quality control of used discs and media was performed with reference strains Escherichia coli (ATCC 25922) and S. aureus (ATCC 25923).

Table 1. Susceptibility table-reference values for Staphylococcus spp.

\begin{tabular}{|c|c|c|c|c|}
\hline \multirow{2}{*}{ Antimicrobials } & \multirow{2}{*}{$\begin{array}{c}\text { Antibiotics Concentration } \\
\text { Per Disc }(\mu \mathrm{g})\end{array}$} & \multicolumn{3}{|c|}{ Diameter (mm) } \\
\hline & & $\mathbf{R}$ & $\mathbf{S}$ & Source \\
\hline Cefoxitin (S. aureus, S. lugdunensis) & 30 & $\leq 21$ & $\geq 22$ & CLSI VET 01 S (2018) \\
\hline Cefoxitin (CNS) & 30 & $\leq 24$ & $\geq 25$ & CLSI VET 01 S (2018) \\
\hline $\begin{array}{l}\text { Oxacillin (S. aureus, } \\
\text { S. pseudintermedius, } \\
\text { Staphylococcus spp.) }\end{array}$ & 5 & $<20$ & $\geq 20$ & $\begin{array}{l}\text { CASFM } \\
(2018)\end{array}$ \\
\hline Amoxicillin/clavulanic acid & $20 / 10$ & $\leq 19$ & $\geq 22$ & NCCLS (2002) \\
\hline $\begin{array}{l}\text { Trimethoprim/sulfamethoxazole } \\
\text { (Staphylococcus spp.) }\end{array}$ & $1.25 / 23.75$ & $\leq 10$ & $\geq 16$ & CLSI VET 01 S (2018) \\
\hline Gentamicin (S. aureus) & 10 & $<18$ & $\geq 18$ & $\begin{array}{l}\text { EUCAST } \\
(2020)\end{array}$ \\
\hline Gentamicin (CNS) & 10 & $<22$ & $\geq 22$ & $\begin{array}{l}\text { EUCAST } \\
(2020)\end{array}$ \\
\hline Tetracycline (Staphylococcus spp.) & 30 & $\leq 17$ & $\geq 23$ & CLSI VET 01 S (2018) \\
\hline Chloramphenicol (Staphylococcus spp.) & 30 & $\leq 12$ & $\geq 18$ & CLSI VET 01 S (2018) \\
\hline Erythromycin (Staphylococcus spp.) & 15 & $\leq 13$ & $\geq 23$ & CLSI VET 01 S (2018) \\
\hline Florfenicol (Staphylococcus spp.) & 30 & $\leq 18$ & $\geq 22$ & CLSI VET 01 (2013) \\
\hline Clindamycin (Staphylococcus spp.) & 2 & $\leq 14$ & $\geq 21$ & CLSI VET 01 S (2018) \\
\hline Enrofloxacin (Staphylococcus spp.) & 5 & $\leq 16$ & $\geq 23$ & CLSI VET 01 S (2018) \\
\hline Nitrofurantoin (Staphylococcus spp.) & 100 & $\leq 14$ & $\geq 17$ & CLSI VET 01 S (2018) \\
\hline Novobiocin & 30 & $\leq 17$ & $\geq 22$ & BD BBL (2020) \\
\hline Rifaximin & 40 & $<10$ & $>19$ & BIOPHARM (2020) \\
\hline
\end{tabular}

\subsection{MRS Molecular Characterization}

In 35 MRS isolates including S. pseudintermedius $(n=20)$, S. aureus $(n=7)$, S. haemolyticus $(n=4), S$. intermedius $(n=2)$, and S. epidermidis $(n=2)$, polymerase chain reaction (PCR) for mecA gene detection was used to confirm methicillin resistance. Eight strains failed to resuscitate, and PCR was not performed. Gene mecA encodes the production of penicillin-binding protein PBP2A (or PBP2') and is considered the gold standard for methicillin resistance determination. For characterization of MRS isolates, the presence of virulence factor genes, including MSCRAMM, biofilm and the main toxin genes, were detected by PCR (polymerase chain reaction). The following genes were targeted: cna (encoding collagen-binding protein), eno (encoding laminin-binding protein), clf $A$ and $c l f B$ (encoding clumping factors A and B), fib (encoding fibrinogen-binding protein), $e b p$ 
(encoding elastin-binding protein), $b b p$ (encoding bone sialoprotein-binding protein), fnbA and $f n b B$ (encoding fibronectin-binding proteins $A$ and $B$ ), $i c a A$ (encoding polysaccharide intercellular adhesin), et $A$ (exfoliative toxin $A$ ), et $B$ (exfoliative toxin B), and tsst (encoding toxic shock syndrometoxin) (see Table 2).

Table 2. Polymerase chain reaction primers used in this study to detect virulence factor genes in methicillin-resistant staphylococci.

\begin{tabular}{|c|c|c|c|c|}
\hline Gene & Primer & Nucleotide Sequence $\left(5^{\prime}-3^{\prime}\right)$ & Amplicon Size & Reference \\
\hline \multirow{2}{*}{ mecA } & MECA-1 & GTAGAAATGACTGAACGTCCGATAA & 310 & [38] \\
\hline & MECA-2 & CСAATTCCACATTGTTTCGGTCTAA & & \\
\hline \multirow{2}{*}{ cna } & CNA-1 & GTCAAGCAGTTATTAACACCAGAC & 423 & [39] \\
\hline & CNA-2 & AATCAGTAATTGCACTTTGTCCACTG & & \\
\hline \multirow{2}{*}{ eno } & ENO-1 & ACGTGCAGCAGCTGACT & 302 & [39] \\
\hline & ENO-2 & CAACAGCATYСTTCAGTAССТTC & & \\
\hline \multirow{2}{*}{ clfA } & CLFA-1 & ATTGGCGTGGCTTCAGTGCT & 292 & [39] \\
\hline & CLFA-2 & CGTTTCTTCCGTAGTTGCATTTG & & \\
\hline \multirow{2}{*}{$c l f B$} & CLFB-1 & ACATCAGTAATAGTAGGGGGCAAC & 205 & [39] \\
\hline & CLFB-2 & TTCGCACTGTTTGTGTTTGCAC & & \\
\hline \multirow{2}{*}{ fib } & FIB-1 & CTACAACTACAATTGCCGTCAACAG & 404 & {$[39]$} \\
\hline & FIB-2 & GCTCTTGTAAGACCATTTTCTTCAC & & \\
\hline \multirow{2}{*}{$e b p$} & EBP-1 & CATCCAGAACCAATCGAAGAC & 186 & [39] \\
\hline & EBP-2 & CTTAACAGTTACATCATCATGTTTATCTTTG & & \\
\hline \multirow{2}{*}{$b b p$} & BBP-1 & AАCTACATCTAGTACTCAACAACAG & 575 & [39] \\
\hline & BBP-2 & ATGTGCTTGAATAACACCATCATCT & & \\
\hline \multirow{2}{*}{$f n b A$} & FNBA-1 & CACAACCAGCAAATATAG & 1362 & [40] \\
\hline & FNBA-2 & CTGTGTGGTAATCAATGTC & & \\
\hline \multirow{2}{*}{$f n b B$} & FNBB-1 & GTAACAGCTAATGGTCGAATTGATACT & 524 & [39] \\
\hline & FNBB-2 & CAAGTTCGATAGGAGTACTATGTTC & & \\
\hline \multirow{2}{*}{$i c a A$} & ICAA-1 & GATTATGTAATGTGCTTGGA & 770 & [40] \\
\hline & ICAA-2 & ACTACTGCTGCGTTAATAAT & & \\
\hline \multirow{2}{*}{ et $A$} & ETA-1 & CTATTTACTGTAGGAGCTAG & 741 & [41] \\
\hline & ETA-2 & ATTTATTTGATGCTCTCTAT & & \\
\hline \multirow{2}{*}{ etB } & ETB-1 & ACGGCTATATACATTCAATT & 200 & [41] \\
\hline & ETB-2 & TCCATCGATAATATACCTAA & & \\
\hline \multirow{2}{*}{ tsst } & TSST-1 & AAGCCCTTTGTTGCTTGCG & 445 & [42] \\
\hline & TSST-2 & ATCGAACTTTGGCCCATACTTT & & \\
\hline
\end{tabular}

Quick boiling method was used for DNA isolation. A number of colonies of pure bacterial culture were resuspended in $50 \mu \mathrm{L}$ of sterile distilled water. The suspension was incubated for $10 \mathrm{~min}$ at $100{ }^{\circ} \mathrm{C}$ and centrifuged for $10 \mathrm{~min}$ at $10,000 \times \mathrm{g}$ at $4{ }^{\circ} \mathrm{C}$. The supernatant was used in the PCR reaction as template DNA. Polymerase chain reactions were performed according to the protocols described in references in Table 2. Fragments were analyzed by electrophoresis in $2 \%$ agarose gel stained with ethidium bromide and visualized under ultraviolet light. Strains used as positive controls were $S$. aureus CCM 2353 for cna, eno, clfA, clfB, fib, ebp, bbp, fnbA, icaA; S. aureus CCM 2773 for fnbB; S. aureus CCM 7056 for et $A$ and etB; and S. aureus RF122 (Fitzgerald J.R., University of Edinburgh, Edinburgh) [43] for tsst gene. 


\section{Results}

Out of 854 (16.37\% prevalence) isolated staphylococci strains, 613 strains belonged to the group of CPSs (11.75\% prevalence) and 241 strains to the group of CNSs (4.62\% prevalence). The detailed numbers and prevalence of different species of staphylococci are shown in Table 3. Of these, 43 strains of 6 species were methicillin-resistant staphylococci (MRS) ( $n=43 ; 0.82 \%$ prevalence). Of them, 36 strains with 4 species ( $n=36 ; 0.69 \%$ prevalence) belonged to the CPS group, and 7 strains of 2 species ( $n=7$; $0.13 \%$ prevalence) to the CNS group. The most prevalent species among the MRS strains were S. pseudintermedius ( $n=24 ; 0.46 \%$ prevalence) and $S$. aureus $(n=7 ; 0.13 \%$ prevalence). Table 4 shows the distribution of individual species of staphylococci in animal population in the observed period. The exact distribution of MRS strains in different species and groups of domestic animals is shown in Table 5. It follows from this table that MRS of all six detected species were isolated from domestic carnivores and their prevalence was the highest in these animals ( $n=34 ; 1.38 \%$ prevalence). Surprisingly, three of the six MRS species were detected in rodents (S. aureus, S. haemolyticus, and S. pseudintermedius) ( $n=3 ; 1.27 \%$ prevalence). In ruminants, pigs, solipeds, and exotic birds, MRS of one species was detected in each $(n=1$, prevalence: $0.16 \%, 1.41 \%, 1.10 \%$, and $0.97 \%$, respectively). Table 6 shows the distribution of MRS isolates in different organs and organ systems. The greatest species diversity and prevalence was found in skin $(n=21 ; 4$ species; $4.79 \%$ prevalence) and the respiratory system ( $n=8 ; 4$ species; $2.72 \%$ prevalence). All MRS strains $(100 \%)$ showed resistance to oxacillin, cephalothin, cefoxitin, cefovecin, piperacillin and amoxicillin/clavulanic acid. Most of these isolates were co-resistant to enrofloxacin (93\%). In contrast, the isolates showed high susceptibility to florfenicol and nitrofurantoin (100\%). Susceptibility to novobiocin and rifaximin was also high ( $97.7 \%$ of susceptible strains). The detailed results are shown in Table 7.

Table 3. Number and prevalence of isolated staphylococci in animals during 1 April 2019-31 May 2020.

\begin{tabular}{|c|c|c|c|c|c|}
\hline $\begin{array}{l}\text { Staphylococcus } \\
\text { Species }\end{array}$ & $\begin{array}{l}\text { Number of } \\
\text { Isolated Strains } \\
\qquad(n=)\end{array}$ & Prevalence (\%) & Staphylococcus Species & $\begin{array}{l}\text { Number of } \\
\text { Isolated Strains } \\
(n=)\end{array}$ & Prevalence $(\%)$ \\
\hline S. aureus & 205 & 3.93 & Mammaliicoccus lentus * & 3 & 0.06 \\
\hline S. arlettae & 3 & 0.06 & S. lugdunensis & 1 & 0.02 \\
\hline S. capitis ssp. capitis & 2 & 0.04 & S. lutrae & 3 & 0.06 \\
\hline S. caprae & 2 & 0.04 & S. petrasii ssp. petrasii ${ }^{*}$ & 1 & 0.02 \\
\hline S. carnosus & 1 & 0.02 & S. pseudintermedius & 336 & 6.44 \\
\hline S. caseolyticus & 1 & 0.02 & S. coagulans * & 19 & 0.36 \\
\hline S. chromogenes & 45 & 0.86 & S. schleiferi ssp. schleiferi ${ }^{*}$ & 2 & 0.04 \\
\hline S. cohnii ssp. cohnii & 1 & 0.02 & Mammaliicoccus sciuri ${ }^{*}$ & 18 & 0.34 \\
\hline S. delphini & 3 & 0.06 & S. simulans & 11 & 0.21 \\
\hline S. epidermidis & 23 & 0.44 & S. succinus ssp. succinus * & 2 & 0.04 \\
\hline S. equorum & 7 & 0.13 & Mammaliicoccus vitulinus * & 1 & 0.02 \\
\hline S. felis & 32 & 0.61 & S. warneri & 2 & 0.04 \\
\hline S. haemolyticus & 68 & 1.30 & S. $x y l o s u s$ & 16 & 0.31 \\
\hline S. hyicus & 6 & 0.11 & & & \\
\hline S. intermedius & 40 & 0.77 & Total & 854 & 16.37 \\
\hline
\end{tabular}


Table 4. Total number of staphylococci strains (by species) isolated from animals during 1 April 2019-31 May 2020.

\begin{tabular}{|c|c|c|c|c|c|c|c|c|c|c|c|c|c|c|c|c|c|c|c|c|c|c|c|c|c|c|c|c|c|}
\hline Staphylococcus spp. & 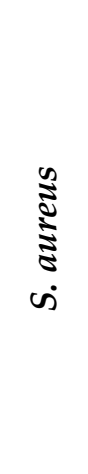 & 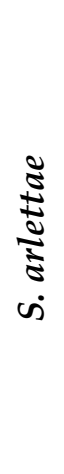 & 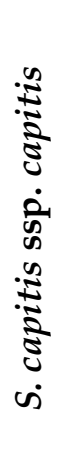 & $\begin{array}{c}\tilde{\Xi} \\
\frac{5}{\delta} \\
\dot{s} \\
\dot{\omega}\end{array}$ & 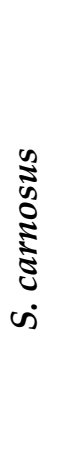 & 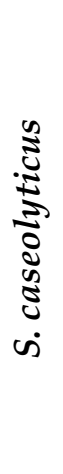 & 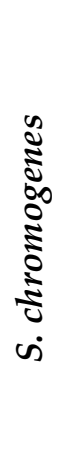 & 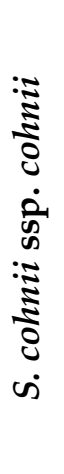 & 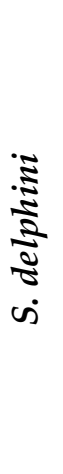 & 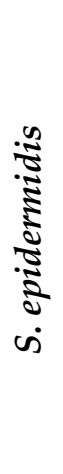 & 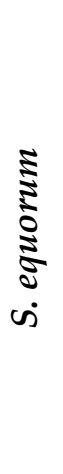 & $\underbrace{\frac{\infty}{0}}_{\dot{0}}$ & 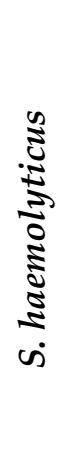 & 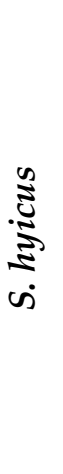 & ஸे & $\begin{array}{l}\frac{*}{0} \\
\frac{\mathfrak{Z}}{\Sigma} \\
\frac{\Xi}{\Sigma} \\
\dot{\Sigma}\end{array}$ & 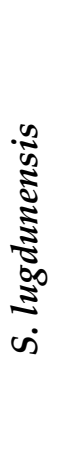 & 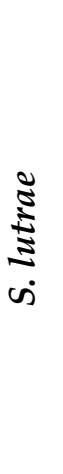 & 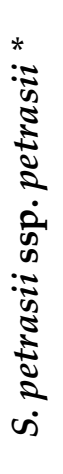 & 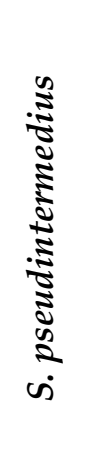 & 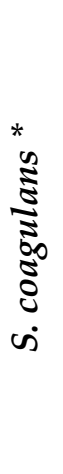 & 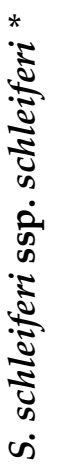 & 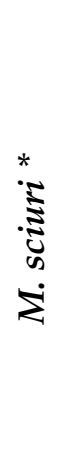 & 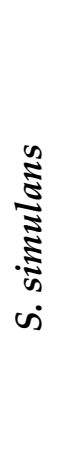 & 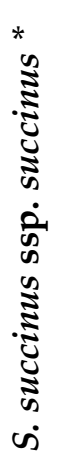 & 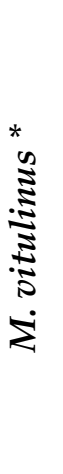 & 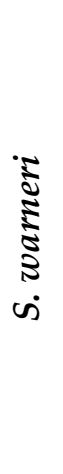 & 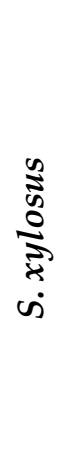 & 胥 \\
\hline $\begin{array}{l}\text { Domestic carnivores } \\
\text { (dogs and cats) }\end{array}$ & 6 & 0 & 2 & 0 & 1 & 0 & 3 & 0 & 1 & 8 & 0 & 32 & 14 & 0 & 38 & 0 & 0 & 2 & 1 & 333 & 11 & 2 & 2 & 1 & 0 & 0 & 1 & 0 & 458 \\
\hline Ruminants & 171 & 3 & 0 & 2 & 0 & 1 & 40 & 1 & 0 & 5 & 1 & 0 & 42 & 1 & 0 & 2 & 0 & 1 & 0 & 0 & 3 & 0 & 10 & 9 & 2 & 1 & 1 & 12 & 308 \\
\hline Pigs & 6 & 0 & 0 & 0 & 0 & 0 & 0 & 0 & 0 & 4 & 0 & 0 & 0 & 5 & 0 & 0 & 0 & 0 & 0 & 0 & 2 & 0 & 0 & 1 & 0 & 0 & 0 & 0 & 18 \\
\hline Solipeds & 12 & 0 & 0 & 0 & 0 & 0 & 2 & 0 & 2 & 0 & 6 & 0 & 0 & 0 & 2 & 1 & 1 & 0 & 0 & 1 & 2 & 0 & 0 & 0 & 0 & 0 & 0 & 0 & 29 \\
\hline Exotic mammals & 1 & 0 & 0 & 0 & 0 & 0 & 0 & 0 & 0 & 2 & 0 & 0 & 0 & 0 & 0 & 0 & 0 & 0 & 0 & 0 & 1 & 0 & 0 & 0 & 0 & 0 & 0 & 0 & 4 \\
\hline Exotic birds & 1 & 0 & 0 & 0 & 0 & 0 & 0 & 0 & 0 & 3 & 0 & 0 & 0 & 0 & 0 & 0 & 0 & 0 & 0 & 0 & 0 & 0 & 0 & 0 & 0 & 0 & 0 & 1 & 5 \\
\hline Rodents & 4 & 0 & 0 & 0 & 0 & 0 & 0 & 0 & 0 & 0 & 0 & 0 & 12 & 0 & 0 & 0 & 0 & 0 & 0 & 2 & 0 & 0 & 6 & 0 & 0 & 0 & 0 & 0 & 24 \\
\hline Total & 205 & 3 & 2 & 2 & 1 & 1 & 45 & 1 & 3 & 23 & 7 & 32 & 68 & 6 & 40 & 3 & 1 & 3 & 1 & 336 & 19 & 2 & 18 & 11 & 2 & 1 & 2 & 16 & 854 \\
\hline
\end{tabular}


Table 5. Number and prevalence (\%) of oxacillin-resistant staphylococci strains isolated from domestic animals during 1 April 2019-31 May 2020.

\begin{tabular}{|c|c|c|c|c|c|c|c|c|}
\hline $\begin{array}{c}\begin{array}{c}\text { Staphylococcus } \\
\text { Species }\end{array} \\
\text { Animal } \\
\text { (Group) }\end{array}$ & S. aureus & S. epidermidis & S. haemolyticus & S. intermedius & $\begin{array}{l}\text { S. pseudinter- } \\
\text { medius }\end{array}$ & S. coagulans & Total & $\begin{array}{c}\text { Number of } \\
\text { Samples }\end{array}$ \\
\hline $\begin{array}{c}\text { Domestic } \\
\text { carnivores } \\
\text { (dogs and cats) }\end{array}$ & $1(0.04)$ & $1(0.04)$ & $4(0.16)$ & $4(0.16)$ & $23(0.93)$ & $1(0.04)$ & $34(1.38)$ & 2471 \\
\hline Ruminants & $3(0.16)$ & 0 & 0 & 0 & 0 & 0 & $3(0.16)$ & 1836 \\
\hline Pigs & $1(1.41)$ & 0 & 0 & 0 & 0 & 0 & $1(1.41)$ & 71 \\
\hline Solipeds & $1(1.10)$ & 0 & 0 & 0 & 0 & 0 & $1(1.10)$ & 91 \\
\hline Birds & 0 & 0 & 0 & 0 & 0 & 0 & 0 & 242 \\
\hline $\begin{array}{l}\text { Exotic } \\
\text { mammals }\end{array}$ & 0 & 0 & 0 & 0 & 0 & 0 & 0 & 84 \\
\hline Exotic birds & 0 & $1(0.97)$ & 0 & 0 & 0 & 0 & $1(0.97)$ & 103 \\
\hline Exotic reptiles & 0 & 0 & 0 & 0 & 0 & 0 & 0 & 46 \\
\hline Fish & 0 & 0 & 0 & 0 & 0 & 0 & 0 & 35 \\
\hline Insects(bees) & 0 & 0 & 0 & 0 & 0 & 0 & 0 & 3 \\
\hline Rodents & $1(0.42)$ & 0 & $1(0.42)$ & 0 & $1(0.42)$ & 0 & $3(1.27)$ & 236 \\
\hline Total & $7(0.13)$ & $2(0.04)$ & $5(0.10)$ & $4(0.08)$ & $24(0.46)$ & $1(0.02)$ & $43(0.82)$ & 5218 \\
\hline
\end{tabular}

Table 6. Number and prevalence (\%) of oxacillin-resistant staphylococci strains isolated from organs of domestic animals during 1 April 2019-31 May 2020.

\begin{tabular}{|c|c|c|c|c|c|c|c|c|}
\hline $\begin{array}{c}\begin{array}{c}\text { Staphylococcus } \\
\text { Species }\end{array} \\
\begin{array}{c}\text { Organ } \\
\text { (Apparatus) }\end{array}\end{array}$ & S. aureus & S. epidermidis & S. haemolyticus & S. intermedius & $\begin{array}{l}\text { S. pseudinter- } \\
\text { medius }\end{array}$ & S. coagulans & Total & $\begin{array}{l}\text { Number of } \\
\text { Samples }\end{array}$ \\
\hline Ear & 0 & 0 & 0 & 0 & $4(0.79)$ & 0 & $4(0.79)$ & 507 \\
\hline Eye & 0 & 0 & 0 & 0 & $2(1.12)$ & 0 & $2(1.12)$ & 179 \\
\hline Skin & $2(0.46)$ & 0 & 0 & $4(0.91)$ & $14(3.20)$ & $1(0.23)$ & $21(4.79)$ & 438 \\
\hline Respiratory & $1(0.34)$ & $1(0.34)$ & $4(1.36)$ & 0 & $2(0.68)$ & 0 & $8(2.72)$ & 294 \\
\hline Digestive & 0 & 0 & 0 & 0 & $2(0.10)$ & 0 & $2(0.10)$ & 1983 \\
\hline $\begin{array}{l}\text { Mammary } \\
\text { gland }\end{array}$ & $3(0.19)$ & 0 & 0 & 0 & 0 & 0 & $3(0.19)$ & 1576 \\
\hline Urogenital & 0 & $1(0.49)$ & $1(0.49)$ & 0 & 0 & 0 & $2(0.97)$ & 206 \\
\hline Musculoskeletal & $1(3.33)$ & 0 & 0 & 0 & 0 & 0 & $1(3.33)$ & 30 \\
\hline Lymphatic & 0 & 0 & 0 & 0 & 0 & 0 & 0 & 2 \\
\hline Circulation & 0 & 0 & 0 & 0 & 0 & 0 & 0 & 3 \\
\hline Nervous & 0 & 0 & 0 & 0 & 0 & 0 & 0 & 0 \\
\hline Total & $7(0.13)$ & $2(0.04)$ & $5(0.10)$ & $4(0.08)$ & $24(0.46)$ & $1(0.02)$ & $43(0.82)$ & 5218 \\
\hline
\end{tabular}

A total of 35 suspected MRS strains (according to disc diffusion method) were characterized by molecular methods, and all were positive for mecA gene. All MRS tested (except of two $S$. aureus isolated from cat urine and cow milk) were positive for eno gene encoding laminin-binding protein. Genes $c n a, c l f A, c l f B$, and $i c a A$ were detected in all of seven $S$. aureus isolates, and $f n b B$ was detected in six $S$. aureus isolates. None of these genes were detected in non-S. aureus isolates. None of MRS tested strain was positive for toxin genes et $A$, et $B$ or tsst (see Table 8 ). 
Table 7. Susceptibility of oxacillin-resistant strains of staphylococci isolated from domestic animals during 1 April 2019-31 May 2020 to antimicrobials (number of susceptible/examined and percentage of susceptible).

\begin{tabular}{|c|c|c|c|c|c|c|c|}
\hline $\begin{array}{c}\text { Staphylococcus } \\
\text { Species }\end{array}$ & S. aureus & S. epidermidis & S. haemolyticus & S. intermedius & $\begin{array}{l}\text { S. pseudinter- } \\
\text { medius }\end{array}$ & S. coagulans & Total \\
\hline Rifaximin & $7 / 7(100 \%)$ & $\begin{array}{c}1 / 2 \\
(50 \%)\end{array}$ & $\begin{array}{c}5 / 5 \\
(100 \%)\end{array}$ & $\begin{array}{c}4 / 4 \\
(100 \%)\end{array}$ & $24 / 24(100 \%)$ & $1 / 1(100 \%)$ & $\begin{array}{c}42 / 43 \\
(97.7 \%)\end{array}$ \\
\hline $\begin{array}{c}\text { Trimethoprim/ } \\
\text { sulphamethoxazole }\end{array}$ & $6 / 7(85.7 \%)$ & $\begin{array}{c}1 / 2 \\
(50 \%)\end{array}$ & $\begin{array}{c}0 / 5 \\
(0 \%)\end{array}$ & $2 / 4(50.0 \%)$ & $11 / 24(45.8 \%)$ & $1 / 1(100 \%)$ & $\begin{array}{c}21 / 43 \\
(48.8 \%)\end{array}$ \\
\hline Gentamicin & $3 / 7(42.9 \%)$ & $\begin{array}{l}0 / 2 \\
(0 \%)\end{array}$ & $\begin{array}{c}0 / 5 \\
(0 \%)\end{array}$ & $\begin{array}{c}0 / 4 \\
(0 \%)\end{array}$ & $5 / 24(20.8 \%)$ & $1 / 1(100 \%)$ & $\begin{array}{c}9 / 43 \\
(20.9 \%)\end{array}$ \\
\hline Tetracycline & $\begin{array}{c}0 / 7 \\
(0 \%)\end{array}$ & $\begin{array}{c}0 / 2 \\
(0 \%)\end{array}$ & $\begin{array}{c}2 / 5 \\
(40.0 \%)\end{array}$ & $1 / 4(25.0 \%)$ & $4 / 24(16.7 \%)$ & $1 / 1(100 \%)$ & $8 / 43(18.6 \%)$ \\
\hline Chloramphenicol & $7 / 7(100 \%)$ & $2 / 2(100 \%)$ & $\begin{array}{c}5 / 5 \\
(100 \%)\end{array}$ & $2 / 4(50.0 \%)$ & $17 / 24(70.8 \%)$ & $1 / 1(100 \%)$ & $\begin{array}{c}34 / 43 \\
(79.1 \%)\end{array}$ \\
\hline Florfenicol & $7 / 7(100 \%)$ & $2 / 2(100 \%)$ & $\begin{array}{c}5 / 5 \\
(100 \%)\end{array}$ & $\begin{array}{c}4 / 4 \\
(100 \%)\end{array}$ & $24 / 24(100 \%)$ & $1 / 1(100 \%)$ & $43 / 43(100 \%)$ \\
\hline Erythromycin & $6 / 7(85.7 \%)$ & $0 / 2(0 \%)$ & $\begin{array}{c}0 / 5 \\
(0 \%)\end{array}$ & $\begin{array}{c}0 / 4 \\
(0 \%)\end{array}$ & $\begin{array}{c}2 / 24 \\
(8.3 \%)\end{array}$ & $1 / 1(100 \%)$ & $\begin{array}{c}9 / 43 \\
(20.9 \%)\end{array}$ \\
\hline Clindamycin & $5 / 7(71.4 \%)$ & $\begin{array}{c}1 / 2 \\
(50 \%)\end{array}$ & $\begin{array}{c}2 / 5 \\
(40.0 \%)\end{array}$ & $\begin{array}{c}0 / 4 \\
(0 \%)\end{array}$ & $\begin{array}{c}2 / 24 \\
(8.3 \%)\end{array}$ & $1 / 1(100 \%)$ & $\begin{array}{c}11 / 43 \\
(25.6 \%)\end{array}$ \\
\hline Enrofloxacin & $\begin{array}{c}3 / 7 \\
(42.9 \%) \\
\end{array}$ & $\begin{array}{c}0 / 2 \\
(0 \%)\end{array}$ & $\begin{array}{c}0 / 5 \\
(0 \%)\end{array}$ & $\begin{array}{l}0 / 4 \\
(0 \%)\end{array}$ & $\begin{array}{l}0 / 24 \\
(0 \%)\end{array}$ & $\begin{array}{c}0 / 1 \\
(0 \%)\end{array}$ & $\begin{array}{c}3 / 43 \\
(7.0 \%)\end{array}$ \\
\hline Novobiocin & $7 / 7(100 \%)$ & $2 / 2(100 \%)$ & $\begin{array}{c}4 / 5 \\
(80.0 \%)\end{array}$ & $\begin{array}{c}4 / 4 \\
(100 \%)\end{array}$ & $24 / 24(100 \%)$ & $1 / 1(100 \%)$ & $\begin{array}{c}42 / 43 \\
(97.7 \%)\end{array}$ \\
\hline Nitrofurantoin & $7 / 7(100 \%)$ & $2 / 2(100 \%)$ & $\begin{array}{c}5 / 5 \\
(100 \%)\end{array}$ & $\begin{array}{c}4 / 4 \\
(100 \%)\end{array}$ & $24 / 24(100 \%)$ & $1 / 1(100 \%)$ & $43 / 43(100 \%)$ \\
\hline
\end{tabular}

Table 8. Occurrence of virulence factor genes in methicillin-resistant Staphylococcus aureus $(n=7)$.

\begin{tabular}{ccccccccc}
\hline Animal & Matter & mecA & cna & eno & clfA & clfB & fnbB & icaA \\
\hline Cat & urine & + & + & - & + & + & - & + \\
Pig & joint & + & + & + & + & + & + & + \\
Cat & skin & + & + & + & + & + & + & + \\
Cow & milk & + & + & + & + & + & + & + \\
Cow & milk & + & + & - & + & + & + & + \\
Horse & skin & + & + & + & + & + & + & + \\
Dog & skin & + & + & + & + & + & + & +
\end{tabular}

mecA-gene encoding methicillin resistance, can-collagen-binding protein gene, eno-laminin-binding protein gene, $c l f A$ and $c l f B$ - genes encoding clumping factors, fib - fibrinogen-binding protein gene, $e b p$ - elastin-binding protein gene, $b b p$ - bone sialoprotein-binding protein gene, $f n b A$ and $f n b B$ - genes encoding fibronectin-binding proteins, ica $A$ - polysaccharide intercellular adhesin gene, $e t A$ and $e t B$-exfoliative toxin genes, tsst-toxic shock syndrome toxin gene.

\section{Discussion}

Even though the time span of collection and testing of clinical samples in our study was rather short, a quite large species diversity of the isolated Staphylococcus strains was shown and confirmed in domestic animals. A total of 28 Staphylococcus species were detected. In our study, of the CPS, the major species identified was S. pseudintermedius ( $n=336 ; 6.44 \%$ prevalence), which was predominantly found in domestic carnivores, especially dogs. The second most frequently encountered CPS was S. aureus $(n=205$; $3.93 \%$ prevalence), surprisingly most often found in ruminants and solipeds, followed by S. intermedius ( $n=40 ; 0.77 \%$ prevalence), which was also predominant in carnivores, especially dogs. Out of the CNS, the major species was S. haemolyticus $(n=68$; $1.30 \%$ prevalence), most often isolated from ruminants and carnivores, followed by S. chromogenes ( $n=45 ; 0.86 \%$ prevalence), which was most prevalent in ruminants, then followed by $S$. felis $(n=32 ; 0.61 \%)$, having a clear affinity for cats, and S. epidermidis ( $n=23 ; 0.44 \%$ prevalence), which was most frequently isolated from clinical material from domestic carnivores, ruminants, and pigs. S. chromogenes has the ability to form biofilms and in veterinary medicine is also a common pathogen of the mammary 
gland of cattle ( $8.8 \%$ to $51.4 \%$ of isolated CNS strains) $[45,46]$. The literature sources confirmed that all the above-mentioned four CNS species may be pathogenic to animals, and some of them also to humans. S. haemolyticus is an opportunistic pathogen infecting debilitated human patients [18] and is often detected in veterinary laboratories in association with mastitis in cattle (12.2\% to $20.3 \%$ of isolated CNS strains) $[45,46]$.

Not surprisingly, most MRS isolates originate in the skin and the respiratory system of animals, which is consistent with the literature. Our findings of MRS strains of staphylococci in domestic animals confirmed that S. aureus, S. pseudintermedius, S. intermedius, S. epidermidis, and S. haemolyticus species may have resistance genes to methicillin, as previously reported by Votava et al. (2006) and Oreiby et al. (2019) [9,23]. In our study, the strain S. coagulans was also detected, which in addition to methicillin resistance also showed resistance to all beta-lactam antibiotics and to enrofloxacin. The prevalence of MRSA strains in our study was relatively low $(n=43 ; 0.82 \%)$ in comparison with $15.5 \%$ in the human clinical material of hospitalized patients [47] and the prevalence of veterinary MRSA strains isolated, for example, from clinical material from domestic carnivores $(16.1 \%)$ in the Czech Republic [12], as well as from milk of cows with signs of mastitis in the Czech Republic and Egypt where the prevalence of MRSA ranged from $31.7 \%$ to 50\% [24,25]. The prevalence of MRS strains of S. pseudintermedius $(n=24 ; 0.46 \%)$ and S. intermedius $(n=4$; $0.08 \%$ ) in our study is also very low in comparison with other Czech studies dealing with S. pseudintermedius (25\%) and S. intermedius (50\%) [12].

The MRS strains of $S$. haemolyticus and S. epidermidis are no exception, with their prevalence in our study of $0.1 \%(n=5)$ and $0.04 \%(n=2)$, respectively, while literature sources reported prevalence, for example in humans, of up to $45.4 \%$ for both of the above bacterial species [48]. This can be attributed to the fact that our strains were collected for 14 months only and that relatively large numbers of various materials from different animals were examined and tested, while other studies focused on narrow spectra of human patients, animals, and clinical materials such as cow's milk samples or clinical specimens from dogs and cats. Our study also shows that the detected strains display a certain species-specificity in terms of antimicrobial resistance.

In addition to beta-lactams, MRS S. aureus (MRSA) strains show 100\% resistance also to tetracycline; S. epidermidis MRS strains also to gentamicin, tetracycline, erythromycin, and enrofloxacin; S. haemolyticus MRS strains also to trimethoprim/sulfamethoxazole, gentamicin, erythromycin, and enrofloxacin; MRS strains of $S$. intermedius also to gentamicin, erythromycin, clindamycin, and enrofloxacin; and MRS strains of S. pseudintermedius and S. coagulans also to enrofloxacin.

Due to the diversity of the clinical material in our study, a diverse capture of adherence factors could be expected, as the first step of successful infection is adherence to different surfaces, depending on the site of infection. However, genes encoding MSCRAMMs were detected only in S. aureus, none in other staphylococci (CNS or CPS).

The exception was the eno gene encoding laminin-binding protein. This gene was detected in all MRS isolated from clinical material except for two S. aureus isolated from cat urine and cow milk. Thus, this virulence factor was shown to be unrelated to the location of the infection, as different isolates from different sites of infection carried this gene. Moreover, other studies described a very common occurrence of the eno gene in both CPS and CNS (73-100\%) [39,49,50]. Consistent with our results, rare occurrences of other MSCRAMM genes in CNS isolates were confirmed by other studies [50]. In contrast, in CPS including S. intermedius, a noticeably higher occurrence was described (ebp-73\%, fib-91\%, and fnbA-7\%) [49]. In our study, neither S. intermedius nor S. pseudintermedius harbored MSCRAMM genes.

Isolates of $S$. aureus from different animals and different sites of infections (cat, pig, cow, horse, dog; urine, joint, milk, skin) were included in our study, yet all these isolates showed very similar MSCRAMM gene profiles: eno+ (except two isolates), $\mathrm{Cna}+$, clfA+, $c l f B+$, and $f n b B+$ (except one isolate from cat urine). 
The icaA gene encoding polysaccharide intercellular adhesin was detected in all MRSA isolates. The ica operon is considered to be the main operon responsible for biofilm formation in the Staphylococcus genus. However, there is not an absolute correlation between the presence or absence of ica genes and biofilm production [51]. In our study, the icaA gene was not detected in other staphylococci (non-S. aureus), consistent with other studies where $i c a A$ was only rarely detected in these strains [51-53]. Genes encoding et $A$, et $B$, and tsst toxins were not detected in our MRS isolates and also in other publications, only the rare occurrence of these genes in staphylococci of animal origin was described $[51,54]$. Although these virulence factors can be found in animal strains, they are generally associated with human clinical pathogens $[51,55]$.

It is well known that the growth of antibiotic resistance is a global problem. Veterinary medicine undoubtedly contributes to its origin and spread due to the application of antimicrobials in water and feed, especially in livestock, and also due to the use of local antibiotics (sprays, suppositories, ointments, dusting powders), where accurate dosing of these substances is not guaranteed. It is therefore necessary to tighten up the legislation governing the use of these substances in animals and to set up a system for its control. At the same time, there is a need to expand and improve university teaching on the use of antimicrobials in the veterinary field.

\section{Conclusions}

The present study demonstrated high prevalence of some CPS and CNS species detected in animals. These strains should be taken seriously from both the epizootiological and epidemiological point of view, as they can pose health risk to both animals and humans not only in terms of potential pathogenicity but in that they can also confer resistance genes and pathogenicity factors to other veterinary and human strains of staphylococci. In terms of antimicrobial susceptibility, it has been confirmed that methicillin-resistant microorganisms are resistant to all other beta-lactam antibiotics, even in isolated veterinary strains and also to amoxicillin/clavulanic acid. All MRS strains of staphylococci isolated in the present study except for three $S$. aureus strains were resistant to enrofloxacin. Due to the occurrence of these microorganisms in farm animals and animals kept for hobby, it would be very appropriate to set up research projects aimed at the detection of oxacillin/methicillinresistant staphylococci in these animal groups and to map their occurrence at least in Europe, because the microecosystems of animals, humans, plants, and the macroecosystem are interconnected. It is therefore necessary to emphasize the issue of correct using of antimicrobials in practice and antibiotic policy in university teaching and to create stricter legislation that would prevent the widespread use of antimicrobials in veterinary medicine, especially in livestock to reduce the emergence and spread of antimicrobial resistance.

Author Contributions: Conceptualization, J.B., V.S., D.S. and M.Z.; methodology, J.B., M.Z., K.N. and O.H.; software, J.B.; validation, O.H., M.Z., D.S.; formal analysis, O.H., V.S.; investigation, J.B., D.S., V.S. and M.Z.; resources, J.B., K.N. and O.H.; data curation, J.B.; writing-original draft preparation, J.B., M.Z., K.N. and O.H.; writing-review and editing, J.B. and O.H.; visualization, J.B., D.S. and V.S.; supervision, O.H.; project administration, M.Z and K.N.; funding acquisition, M.Z. All authors have read and agreed to the published version of the manuscript.

Funding: Supported bythe Ministry of Agriculture of the Czech Republic (Institutional Support No. MZE-RO0518) and by the National Agency for Agricultural Research (Projects No.QK1910212).

Institutional Review Board Statement: Not applicable. All animals were patients, not experimental animals.

Informed Consent Statement: Not applicable. Our study is strictly anonymous and the rights of the animal patients and their owners have not beenviolated in any way.All laboratory tests and data processing were performed on request and with the express consent of the animal owners. 
Data Availability Statement: All data supporting reported results of this work are archived in the personal database of the first author (Bzdil, J.) and partial data concerning the genomic characterization of bacterial strains are stored in the database of the Veterinary Research Institute Brno (Czech Republic).

Acknowledgments: The authors wish to thank Ivana Kucharovičová from the State Veterinary Institute in Jihlava, Romana Beránková from BIOPHARM Research Institute of Biopharmacy and Veterinary Drugs Czech Republic, and Lucie Pokludová from the Institute for State Control of Veterinary Biologicals and Medicines Brno for consultations and valuable information on the susceptibility of staphylococci to certain antimicrobials. The authors also thank Petr Petrás from the National Reference Laboratory for Staphylococci of the National Institute of Public Health in Prague for his help in typing of isolated strains and valuable professional advice. This study was supported by the Ministry of Agriculture of the Czech Republic by grant project NAZV QK1910212 and MZE-RO0518.

Conflicts of Interest: The authors declare no conflict of interest.

\section{References}

1. Julák, J. Introduction to Medical Microbiology, 2nd ed.; Karolinum: Prague, Czech Republic, 2015; p. 404.

2. Bzdil, J.; Holy, O.; Chmelar, D. Gram-positive aerobic and microaerophilic microorganisms isolated from pathological processes and lesions of horses. Vet. Med. 2017, 62, 1-9. [CrossRef]

3. Rossi, C.C.; Dias, I.D.S.; Muniz, I.M.; Lilenbaum, W.; Giambiagi-Demarval, M. The oral microbiota of domestic cats harbors a wide variety of Staphylococcus species with zoonotic potential. Vet. Microbiol. 2017, 201, 136-140. [CrossRef] [PubMed]

4. Mama, O.M.; Gómez-Sanz, E.; Ruiz-Ripa, L.; Gómez, P.; Torres, C. Diversity of staphylococcal species in food producing animals in Spain, with detection of PVL-positive MRSA ST8 (USA300). Vet. Microbiol. 2019, 233, 5-10. [CrossRef] [PubMed]

5. Vasileiou, N.G.C.; Chatzopoulos, D.C.; Sarrou, S.; Fragkou, I.A.; Katsafadou, A.I.; Mavrogianni, V.S.; Petinaki, E.; Fthenakis, G.C. Role of staphylococci in mastitis in sheep. J. Dairy Res. 2019, 86, 254-266. [CrossRef]

6. Quinn, P.J.; Markey, B.K.; Leonard, F.C.; Fitzpatrick, E.S.; Fanning, S.; Hartigan, P.J. Veterinary Microbiology and Microbial Disease, 2nd ed.; Blackwell Publishing: Oxford, UK, 2011; p. 912.

7. Anderson, K.L.; Kearns, R.; Lyman, R.; Correa, M.T. Staphylococci in dairy goats and human milkers, and the relationship with herd management practices. Small Rumin. Res. 2018, 171, 13-22. [CrossRef]

8. Magleby, R.; Bemis, D.A.; Kim, D.; Carroll, K.C.; Castanheira, M.; Kania, S.A.; Jenkins, S.G.; Westblade, L.F. First reported human isolation of Staphylococcus delphini. Diagn. Microbiol. Infect. Dis. 2019, 94, 274-276. [CrossRef] [PubMed]

9. Votava, M.; Černohorská, L.; Heroldová, M.; Holá, V.; Mejzlíková, L.; Ondrovčík, P.; Růžička, F.; Dvořáčková, M.; Woznicová, V.; Zahradníček, O. Special Medical Microbiology; Neptun: Brno, Czech Republic, 2006; p. 495. ISBN 80-902896-6-5. (In Czech)

10. Bradley, A. Bovine Mastitis: An Evolving Disease. Vet. J. 2002, 164, 116-128. [CrossRef]

11. Zecca, E.; Costanzo, M.; Croce, A.; Sola, D.; Pirovano, A.; Matino, E.; Pirisi, M. First reported human case of meningitis by Staphylococcus condimenti. Infection 2019, 47, 651-653. [CrossRef]

12. Bzdil, J.; Štanclová, D.; Toporčák, J.; Dopitová, Š.; Axmannová, M. Staphylococci Isolated from Dogs and Cats and its Susceptibility to Antimicrobials. Veterinářství 2019, 69, 155-160. (In Czech)

13. Songer, J.G.; Post, K.W. Veterinary Microbiology Bacterial and Fungal Agents of Animal Disease; Elsevier Sounders: Philadelphia, PA, USA, 2005; p. 434.

14. Murugaiyan, J.; Walther, B.; Stamm, I.; Abou-Elnaga, Y.; Brueggemann-Schwarze, S.; Vincze, S.; Wieler, L.H.; Lübke-Becker, A.; Semmler, T.; Roesler, U. Species differentiation within the Staphylococcus intermedius group using a refined MALDI-TOF MS database. Clin. Microbiol. Infect. 2014, 20, 1007-1014. [CrossRef]

15. Litster, A.; Moss, S.M.; Honnery, M.; Rees, B.; Trott, D.J. Prevalence of bacterial species in cats with clinical signs of lower urinary tract disease: Recognition of Staphylococcus felis as a possible feline urinary tract pathogen. Vet. Microbiol. 2007, 121, 182-188. [CrossRef]

16. Ahmad, N.I.; Yean, C.Y.; Foo, P.C.; Safiee, A.W.M.; Hassan, S.A. Prevalence and association of Panton-Valentine Leukocidin gene with the risk of sepsis in patients infected with Methicillin Resistant Staphylococcus aureus. J. Infect. Public Health 2020, 13, 1508-1512. [CrossRef]

17. Von Eiff, C.; Peters, G.; Heilmann, C. Pathogenesis of infections due to coagulase-negative staphylococci. Lancet Infect. Dis. 2002, 2, 677-685. [CrossRef]

18. Takeuchi, F.; Watanabe, S.; Baba, T.; Yuzawa, H.; Ito, T.; Morimoto, Y.; Kuroda, M.; Cui, L.; Takahashi, M.; Ankai, A.; et al. Whole-Genome Sequencing of Staphylococcus haemolyticus Uncovers the Extreme Plasticity of Its Genome and the Evolution of Human-Colonizing Staphylococcal Species. J. Bacteriol. 2005, 187, 7292-7308. [CrossRef] [PubMed]

19. Kmieciak, W.; Szewczyk, E.M. Are zoonotic Staphylococcus pseudintermedius strains a growing threat for humans? Folia Microbiol. 2018, 63, 743-747. [CrossRef]

20. Kizerwetter-Świda, M.; Pławińska-Czarnak, J. Staphylococci isolated from animals as a source of genes that confer multidrug resistance to antimicrobial agents of critical importance to public health. Med. Weter. 2017, 73, 626-631. [CrossRef] 
21. Ouoba, L.I.I.; Mbozo, A.B.V.; Anyogu, A.; Obioha, P.I.; Lingani-Sawadogo, H.; Sutherland, J.P.; Jespersen, L.; Ghoddusi, H.B. Environmental heterogeneity of Staphylococcus species from alkaline fermented foods and associated toxins and antimicrobial resistance genetic elements. Int. J. Food Microbiol. 2019, 311, 108356. [CrossRef]

22. Becker, K.; Ballhausen, B.; Köck, R.; Kriegeskorte, A. Methicillin resistance in Staphylococcus isolates: The "mec alphabet" with specific consideration of mecC, a mec homolog associated with zoonotic S. aureus lineages. Int. J. Med Microbiol. 2014, 304, 794-804. [CrossRef] [PubMed]

23. Oreiby, A.; Khalifa, H.; Eld, A.; Ahmed, A.; Shimamoto, T. Clinical and molecular characterization of both methicillin-resistant and-sensitive Staphylococcus aureus mastitis. J. Hell. Vet. Med. Soc. 2019, 70, 1743-1748. [CrossRef]

24. Bzdil, J.; Chaloupka, O.; Bezrouk, Z. Staphylococcus aureus and Bovine Mastitis, Changes in Prevalence and Susceptibility to Antimicrobials in 2007-2016. Veterinářství 2017, 6, 466-471. (In Czech)

25. Ahmed, W.; Neubauer, H.; Tomaso, H.; El Hofy, F.I.; Monecke, S.; Abdeltawab, A.A.; Hotzel, H. Characterization of Staphylococci and Streptococci Isolated from Milk of Bovides with Mastitis in Egypt. Pathogens 2020, 9, 381. [CrossRef]

26. Gordon, R.J.; Lowy, F.D. Pathogenesis of methicillin-resistant Staphylococcus aureus infection. Clin. Infect. Dis. 2008, 46, S350-S359. [CrossRef]

27. Foster, T.J.; Höök, M. Surface protein adhesins of Staphylococcus aureus. Trends Microbiol. 1998, 6, 484-488. [CrossRef]

28. Balachandran, M.; Bemis, D.A.; Kania, S.A. Expression and function of protein A in Staphylococcus pseudintermedius. Virulence 2018, 9, 390-401. [CrossRef] [PubMed]

29. Costerton, J.W.; Stewart, P.S.; Greenberg, E.P. Bacterial Biofilms: A Common Cause of Persistent Infections. Science 1999, 284, 1318-1322. [CrossRef] [PubMed]

30. Oliveira, D.; Borges, A.; Simões, M. Staphylococcus aureus Toxins and Their Molecular Activity in Infectious Diseases. Toxins 2018, 10, 252. [CrossRef]

31. Clinical and Laboratory Standards Institute (CLSI). Performance Standards for Antimicrobial Disk and Dilution Susceptibility Tests for Bacteria Isolated from Animals, Approved Standard, CLSI Document VET01-A4, 4th ed.; Clinical and Laboratory Standards Institute: Wayne, PA, USA, 2013; p. 70.

32. Clinical and Laboratory Standards Institute (CLSI). Performance Standards for Antimicrobial Disk and Dilution Susceptibility Tests for Bacteria Isolated from Animals, CLSI Supplement VET08, 4th ed.; Clinical and Laboratory Standards Institute: Wayne, PA, USA, 2018; p. 170.

33. NCCLS. Performance Standards for Antimicrobial Disk and Dilution Susceptibility Tests for Bacteria Isolated from Animals; Approved Standard, NCCLS Document M31-A2, 2nd ed.; NCCLS: Wayne, PA, USA, 2002; ISBN 1-56238-461-9.

34. European Committee on Antimicrobial Susceptibility Testing (EUCAST). Breakpoint Tables for Interpretation of MICs and Zone Diameters. Version 10.0, 2018; European Committee on Antimicrobial Susceptibility Testing: Växjö, Sweden, 2020. Available online: http:/ / www.eucast.org/clinical_breakpoints (accessed on 25 August 2020).

35. Comité de l'Antibiogramme de la Société Francaise de Microbiologie (CA-SFM). RecommandationsVétérinaries; Société Francaise de Microbiologie: Paris, France, 2018; p. 15.

36. BD BBL. BBL Sensi-Disc Antimicrobial Susceptibility Test Discs, 8840621JAA(02)2014-03; BBL: Le Pont de Claix, France, 2020; p. 15. Available online: https:/ / docplayer.cz/46964153-Bbl-sensi-disc-antimicrobial-susceptibility-test-discs.html (accessed on 24 August 2020).

37. Biopharm. Diagnostics Disc for Susceptibility Testing to Antibiotic Rifaximin; Biopharmacy and Veterinary Drugs Research Institute: Jílové u Prahy, Czech Republic, 2020; p. 1.

38. Geha, D.J.; Uhl, J.R.; Gustaferro, C.A.; Persing, D.H. Multiplex PCR for identification of methicillin-resistant Staphylococci in the clinical laboratory. J. Clin. Microbiol. 1994, 32, 1768-1772. [CrossRef]

39. Tristan, A.; Ying, L.; Bes, M.; Etienne, J.; Vandenesch, F.; Lina, G. Use of Multiplex PCR To Identify Staphylococcus aureus Adhesins Involved in Human Hematogenous Infections. J. Clin. Microbiol. 2003, 41, 4465-4467. [CrossRef]

40. Peacock, S.J.; Moore, C.; Justice, A.; Kantzanou, M.; Story, L.; Mackie, K.; O’Neill, G.; Day, N.P.J. Virulent Combinations of Adhesin and Toxin Genes in Natural Populations of Staphylococcus aureus. Infect. Immun. 2002, 70, 4987-4996. [CrossRef]

41. Růžičková, V.; Voller, J.; Pantůček, R.; Petráš, P.; Doškař, J. Multiplex PCR for detection of three exfoliative toxin serotype genes in Staphylococcus aureus. Folia Microbiol. 2005, 50, 499-502. [CrossRef]

42. Becker, K.; Roth, R.; Peters, G. Rapid and Specific Detection of Toxigenic Staphylococcus aureus: Use of Two Multiplex PCR Enzyme Immunoassays for Amplification and Hybridization of Staphylococcal Enterotoxin Genes, Exfoliative Toxin Genes, and Toxic Shock Syndrome Toxin 1 Gene. J. Clin. Microbiol. 1998, 36, 2548-2553. [CrossRef]

43. Fitzgerald, J.R.; Monday, S.R.; Foster, T.J.; Bohach, G.A.; Hartigan, P.J.; Meaney, W.J.; Smyth, C.J. Characterization of a Putative Pathogenicity Island from Bovine Staphylococcus aureus Encoding Multiple Superantigens. J. Bacteriol. 2001, 183, 63-70, Erratum in J. Bacteriol. 2001, 166, 4259. [CrossRef]

44. Madhaiyan, M.; Wirth, J.S.; Saravanan, V.S. Phylogenomic analyses of the Staphylococcaceae family suggest the reclassification of five species within the genus Staphylococcus as heterotypic synonyms, the promotion of five subspecies to novel species, the taxonomic reassignment of five Staphylococcus species to Mammaliicoccus gen. nov., and the formal assignment of Nosocomiicoccus to the family Staphylococcaceae. Int. J. Syst. Evol. Microbiol. 2020, 70, 5926-5936.

45. Malinowski, E.; Lassa, H.; Kłlossowska, A.; Smulski, S.; Markiewicz, H.; Kaczmarowski, M. Etiological agents of dairy cows mastitis in western part of Poland. Pol. J. Vet. Sci. 2006, 9, 191-194. [PubMed] 
46. Bzdil, J. Spectrum of Aerobic Microorganisms Isolated from Cattle Milk Samples with Symptoms of Mastitis. Veterinářství 2015, 8, 630-635. (In Czech)

47. Silva, V.; Hermenegildo, S.; Ferreira, C.; Manaia, C.; Capita, R.; Alonso-Calleja, C.; Carvalho, I.; Pereira, J.; Maltez, L.; Capelo, J.; et al. Genetic Characterization of Methicillin-Resistant Staphylococcus aureus Isolates from Human Bloodstream Infections: Detection of $\mathrm{MLS}_{\mathrm{B}}$ Resistance. Antibiotics 2020, 9, 375. [CrossRef] [PubMed]

48. Montazeri, E.A.; Seyed-Mohammadi, S.; Dezfuli, A.A.; Khosravi, A.D.; Dastoorpoor, M.; Roointan, M.; Saki, M. Investigation of SCCmec types I-IV in clinical isolates of methicillin-resistant coagulase-negative staphylococci in Ahvaz, Southwest Iran. Biosci. Rep. 2020, 40, BSR20200847. [CrossRef]

49. Salaberry, S.R.S.; Saidenberg, A.; Zuniga, E.; Melville, P.A.; Santos, F.G.B.; Guimarães, E.C.; Gregori, F.; Benites, N.R. Virulence factors genes of Staphylococcus spp. isolated from caprine subclinical mastitis. Microb. Pathog. 2015, 85, 35-39. [CrossRef]

50. Simojoki, H.; Hyvönen, P.; Ferrer, C.P.; Taponen, S.; Pyörälä, S. Is the biofilm formation and slime producing ability of coagulasenegative staphylococci associated with the persistence and severity of intramammary infection? Vet. Microbiol. 2012, 158, 344-352. [CrossRef]

51. Bertelloni, F.; Cagnoli, G.; Ebani, V.V. Virulence and Antimicrobial Resistance in Canine Staphylococcus spp. Isolates. Microorganisms 2021, 9, 515. [CrossRef]

52. Soumya, K.R.; Philip, S.; Sugathan, S.; Mathew, J.; Radhakrishnan, E.K. Virulence factors associated with Coagulase Negative Staphylococci isolated from human infections. 3 Biotech 2017, 7, 140. [CrossRef]

53. Piessens, V.; De Vliegher, S.; Verbist, B.; Braem, G.; Van Nuffel, A.; De Vuyst, L.; Heyndrickx, M.; Van Coillie, E. Characterization of coagulase-negative staphylococcus species from cows' milk and environment based on bap, icaA, and mecA genes and phenotypic susceptibility to antimicrobials and teat dips. J. Dairy Sci. 2012, 95, 7027-7038. [CrossRef] [PubMed]

54. Abdel-Moein, K.A.; Zaher, H.M. The Nasal Carriage of Coagulase-Negative Staphylococci Among Animals and Its Public Health Implication. Vector-Borne Zoonotic Dis. 2020, 20, 897-902. [CrossRef] [PubMed]

55. Adame-Gómez, R.; Castro-Alarcón, N.; Vences-Velázquez, A.; Toribio-Jiménez, J.; Pérez-Valdespino, A.; Leyva-Vázquez, M.A.; Ramírez-Peralta, A. Genetic diversity and virulence factors of S. aureus isolated from food, humans and animals. Int. J. Microbiol. 2020, 2020, 1048097. [CrossRef] [PubMed] 